\title{
BMJ Open Outcomes associated with peripherally inserted central catheters in hospitalised children: a retrospective 7-year single- centre experience
}

\author{
Aditya Badheka, ${ }^{1}$ Jodi Bloxham, ${ }^{1}$ April Schmitz, ${ }^{1}$ Barbara Freyenberger, ${ }^{1}$ \\ Tong Wang, ${ }^{2}$ Sankeerth Rampa, ${ }^{3}$ Jennifer Turi, ${ }^{4}$ Veerasathpurush Allareddy, ${ }^{5}$ \\ Marcelo Auslender, ${ }^{1}$ Veerajalandhar Allareddy ${ }^{1}$
}

To cite: Badheka A, Bloxham J, Schmitz A, et al. Outcomes associated with peripherally inserted central catheters in hospitalised children: a retrospective 7-year singlecentre experience. BMJ Open 2019;9:e026031. doi:10.1136/ bmjopen-2018-026031

- Prepublication history and additional material for this paper are available online. To view these files, please visit the journal online (http://dx.doi org/10.1136/bmjopen-2018026031).

Limited results of this study were presented at the 47 th Annual Congress, Society of critical care medicine. Four abstracts pertaining to this study were presented. One of the abstract was selected and presented as the STAR Research Award.

$A B$ and $J B$ are co-first authors

Received 16 August 2018

Revised 24 June 2019

Accepted 11 July 2019

Check for updates

(C) Author(s) (or their employer(s)) 2019. Re-use permitted under CC BY-NC. No commercial re-use. See rights and permissions. Published by BMJ.

For numbered affiliations see end of article.

Correspondence to Dr Aditya Badheka; aditya-badheka@uiowa.edu

\section{ABSTRACT}

Objectives The use of peripherally inserted central catheters (PICCs) are an integral part of caring for hospitalised children. We sought to estimate the incidence of and identify the risk factors for complications associated with PICCs in an advanced registered nurse practitioners (ARNP)-driven programme.

Design Retrospective cohort study.

Setting Single-centre, large quaternary children's hospital.

Participants Hospitalised children who had PICC inserted from 1 January 2010 to 31 December 2016.

Interventions None.

Measurement and main results A total of 2558 PICCs were placed during the study period. Mean age at PICC insertion was 8.7 years, mean dwell time was 17.7 days. The majority of PICCs $(97.8 \%)$ were placed by ARNP. Most were placed in a single attempt (79.6\%). Mean PICC residual external length outside was $2.1 \pm 2.7 \mathrm{~cm}$. The rate of central line-associated bloodstream infection (CLABSI), thrombosis and significant bleeding were $1.9 \%, 1 \%$ and $0.2 \%$, respectively. The CLABSI rate in infants and early childhood was higher than those aged $\geq 5$ years $(2.8 \%$, $3.1 \%$, respectively vs $1.3 \%$ ). In a multivariate analysis after adjustment of confounding effects of race and gender, infants $(\mathrm{OR}=2.24, \mathrm{Cl}=1.14$ to $4.39, \mathrm{p}=0.02)$ and early childhood cohort $(\mathrm{OR}=2.37, \mathrm{Cl}=1.12$ to $5.01, \mathrm{p}=0.02$ ) were associated with significantly higher odds of developing CLABSI compared with $\geq 5$ years old. In the early childhood cohort, PICCs with longer residual external catheter length $(\mathrm{OR}=1.30,95 \% \mathrm{Cl}=1.07$ to $1.57, \mathrm{p}=0.008)$ and those placed in the operating room $(0 \mathrm{R}=5.49,95 \% \mathrm{Cl}=1.03$ to $29.19, p=0.04$ ), were associated with significantly greater risk of developing CLABSI.

Conclusions The majority of PICCs were successfully placed by ARNPs on the first attempt and had a low incidence of complications. Infants required more attempts for successful PICC placement than older children. The presence of residual external catheter length and placement in the operating room were independent predictors of CLABSI in younger children.

\section{BACKGROUND}

Peripherally inserted central catheters (PICCs) are frequently used as stable
Strengths and limitations of this study

- This is a large study that included multiple ( $>70$ variables) patient, provider and peripherally inserted central catheter (PICC)-related variables in more than 2500 PICCs.

- We minimised the impact of the provider, unit and institutional variances by excluding PICCs that were (1) placed in neonatal intensive care unit by their providers, (2) placed at an outside institution prior to transfer or (3) those that remained in place at discharge from hospital.

- Formal, standardised central line catheter insertion and maintenance bundles were in place throughout the period of study and were strictly adhered to.

- This is a retrospective study that is based on availability of data elements and with no ability to adjust for potential other confounding variables.

intravenous access to deliver nutrition, medications and fluids. ${ }^{1}$ The utilisation of PICCs is increasing in hospitalised children secondary to the ease of bedside insertion, lower risk of injury during placement (ie, pneumothorax, haemothorax, vascular injury) and perceived decreased complication profile when compared with central venous catheters (CVCs). ${ }^{23}$ While PICCs can remain in place for prolonged periods of time, mechanical and infectious complications, such as central line-associated bloodstream infections (CLABSIs), thrombosis and acute bleeding, can occur during the placement or maintenance of PICCs. ${ }^{14}$

CLABSIs are the most common healthcare-associated infection and are associated with high likelihood of morbidity and mortality in critically ill children. ${ }^{5}$ Interventions such as the introduction of insertion bundles has reduced CLABSI rates significantly. ${ }^{56}$ However, despite this, approximately 
30000 cases of CLABSIs occur annually in the USA which may indicate that CLABSIs primarily occur during catheter maintenance. ${ }^{7}$ Current literature suggests that the risk of CLABSI increases with prolonged catheter dwell time, potentially via catheter colonisation and biofilm formation. ${ }^{8}$ This suggests that modifiable risk factors for CLABSIs may be present during the period of catheter maintenance. ${ }^{14910}$

PICC-related thrombosis is a risk factor for catheter malfunction, sepsis secondary to bacterial colonisation of the clot, embolism, recurrent venous thrombosis and loss of intravenous access. ${ }^{11}$ The incidence of PICC-related symptomatic thrombosis ranges from $3 \%$ to $20 \%$, while reported asymptomatic thrombosis rate may be as high as $61 \%{ }^{12}{ }^{13}$ Several studies suggest an association between larger PICC diameter and higher rate of thrombosis. ${ }^{12} 14$ Other risk factors include procoagulant state secondary to malignancy, sepsis and critically illness ${ }^{1516}$ as well as the number of attempts to access the vessel. Fewer attempts to place intravenous access result in lower risk of vessel injury and subsequent thrombus formation. ${ }^{17}$ In addition, fewer attempts required to successfully place intravenous access have been shown to increase patient satisfaction. ${ }^{18}$ Successful placement and maintenance of PICCs depends on a variety of patient, provider and catheter-related factors. Understanding these can help augment the success of paediatric vascular access programme.

Our overall goal was to identify outcomes associated with PICC placement in hospitalised children by our vascular access advanced registered nurse practitioner (ARNP)-driven PICC programme. We sought to find an association between complications and a combination of patient, provider and PICC-related factors. We hypothesised that younger age would be an independent predictor of CLABSI development in hospitalised children with a PICC. Secondarily, we hypothesised that younger age would be an independent predictor for increase in number of sites attempted for successful PICC placement in hospitalised children.

\section{MATERIALS AND METHODS Design, setting and timeframe}

We performed a retrospective analysis of a large cohort of hospitalised children who underwent PICC placement at Stead Family Children's Hospital (SFCH), University of Iowa Hospitals and Clinics from 1 January 2010 to 31 December 2016. SFCH is an urban, academic, paediatric, quaternary referral centre with 190 inpatient beds. We care for all cohorts that require admission irrespective of indication-either primary medical or surgical. The per cent of primary medical versus surgical indication for admission has varied in our institute over time. The surgical patients include all service lines such as cardiac, neuro, orthopaedics, ear, nose and throat, ophthalmology, urology and general surgical patients. Children aged less than or equal to 21 years of age at the time of admission and who had a PICC placed during their hospitalisation were eligible for our study. Children who were primarily admitted to the neonatal intensive care unit or newborn nursery were excluded. Those children who were transferred to our hospital from an outside facility with a PICC in place were likewise excluded. This study was approved by the institutional review board of the University of Iowa.

In year 2010, the vascular access ARNP-driven PICC programme was formalised. All requests for paediatric PICC placement were initially evaluated by the designated vascular access ARNP team. If PICC placement was unsuccessful, it was then attempted by interventional radiology, paediatric cardiac catheterisation laboratory or paediatric surgery for alternative vascular access options. The ARNP team has used ultrasound to facilitate PICC placement since the inception of the formalised programme. PICC insertion and maintenance practices were not changed during the study period.

The data were curated from our electronic health records (EPIC, Electronic Health Records) by three authors (JB, AS, AB). All patient identifiers were removed prior to analysis.

\section{Predictor variables}

Patient variables collected included demographics such as age, race, ethnicity, gender, weight and height at the time of PICC placement. The indication for PICC placement and the primary diagnosis of the hospital admission were also collected.

PICC-related characteristics included the number of lumens, size, length of the catheter inside the patient (internal length) and external length of residual catheter outside the patient measured in centimetres. The primary providers placing the PICCs were the ARNPs. Other providers placing PICCs included paediatric critical care fellows, interventional radiologists, interventional cardiologists, surgeons and paediatric critical care intensivists. The location where the procedure was performed included the paediatric intensive care unit (PICU), operating room, inpatient general paediatric floor, paediatric specialty clinic, cardiac catheterisation laboratory, radiology suite and burn unit. PICC tip location was confirmed by plain radiography and included central (superior vena cava (SVC)/Right Atrial junction or inferior vena cava (IVC)) and peripheral positioning. The number of radiographs performed to confirm positioning was collected. The radiologist formal report was considered the standard for central position determination. PICC insertion site such as scalp, extremity (upper or lower), laterality (left or right) and use of ultrasound was collected. The use of sedation, paralysis, and/or topical anaesthetic during the procedure was also collected. We also documented the presence of concomitant CVCs.

\section{Outcome variables}

The primary outcome variable was complication risk. For the purpose of this study, complications were defined a priori (author consensus and literature review) as three distinct objective variables-CLABSI, bleeding and 
thrombosis. CLABSIs were diagnosed per The Centers for Disease Control and Prevention (CDC) definition and confirmed by the hospital epidemiology division. Significant bleeding was defined by anaemia due to acute blood loss with the need for a transfusion of packed red blood cells or other blood products (fresh frozen plasma or platelets) within 6 hours after placement of the PICC. Extensive chart review pertaining to the blood transfusion event was further assessed to ascertain if there was preprocedural decision to transfuse the blood product. Thrombosis secondary to PICG placement was defined by the presence of thrombus in the vessel where a PICC had been placed. A thrombus was confirmed by vascular ultrasound that was obtained due to the bedside clinician's suspicion for a clot (limb swelling, discoloration, loss of pulses and other concerns) and was confirmed by a radiology attendant. We assessed for pre-existing thrombus by review of any vascular ultrasounds obtained up to 3 months prior to PICC placement. Other outcome variables of interest included the number of attempts for successful PICC placement.

\section{Statistical analysis}

Simple descriptive statistics were used to summarise the data.

In the present study, the primary outcome of interest was development of CLABSI. The primary hypothesis examined was if age was associated with development of CLABSI. Age was categorised into three groups: infancy (up to 12 months), early childhood (13-59 months) and $>5$ years. A multivariable logistic regression model was used to examine the association between age and odds of developing a CLABSI. The effects of sex and race were adjusted in the logistic regression model. Several subset analyses were also conducted to examine outcomes within different age cohort. Bivariate logistic regression analyses were conducted to examine the independent association of each independent variable with the odds of developing a CLABSI. Following this, a mix of patient/PICC line-related variables were included in the multivariable models depending on best fit and availability of adequate number of cases within each level of independent variable.

In the first subset analysis, we examined outcomes within the infant cohort. In this cohort, we examined a mix of patient and PICC line-related variables on the odds of developing CLABSI. The independent variables of included in this model were: age, sex, race, surgical cohort, cardiac cohort, days (dwell time of PICC line), concomitant CVC presence, size of lumen(s), type of lumen, extremity/scalp site, number of times PICC line was attempted, length of PICC line outside (residual catheter external to skin) and length of PICC line inside (internal to the skin). A multivariable logistic regression model was used to examine the simultaneous association between all independent variables and odds of developing a CLABSI.

In the second subset analysis, we examined the factors associated with odds of developing CLABSI in the early childhood cohort using a multivariable logistic regression model. In this model, the independent variables included were: age, sex, race, surgical cohort, cardiac cohort, days (dwell time of PICC line), associated CVC presence, size of lumen(s), use of ultrasound, number of attempts at PICC placement, length of PICC line external to the skin and internal length of PICC line.

The second hypothesis examined in the present study was number of attempts made to obtain a successful PICC line. The primary independent variable was age. Age was divided into the following cohorts: infancy (0-12 months); early childhood (13-59 months); middle childhood (60-131 months); early adolescence (132-179 months); middle adolescence (180-215 months) and late adolescence (216-251 months). The covariates adjusted included: sex, race, place, CVC presence, use of Ultrasound (USG), use of topical, sedation, paralysis, surgical cohort and cardiac cohort. A multivariable linear regression model was used to examine the association between the above-mentioned variables and number of attempts to obtain a successful PICC line.

All multivariable logistic regression models were fit using the maximum likelihood methods. The multivariable linear regression model was fit using the ordinary least squares estimation approach. All statistical analyses were conducted using SPSS V.25.0 software (IBM Corp).

\section{Patient and public involvement}

In this retrospective study, we accessed patient's electronic data from the health records at our institution. All patient identifiers were removed before the analysis was conducted. There was no direct patient and public involvement.

\section{RESULTS}

\section{Characteristics of children who received the PICCs}

During the study period from 2010 to 2016, a total of 2558 PICCs were placed in children. PICC placement annually ranged from 312 in the year 2016 to 393 in the year 2011. The majority of PICCs were placed in Caucasians $80.2 \%$, and gender distribution was equal. (see online supplementary table 1) We divided our cohort into the following subgroups based on age to better define the impact of age on outcomes: infancy (birth $<1$ year), early childhood (1-5 years), middle childhood (5-11 years), early adolescence (11-15 years), middle adolescence (15-18 years) and late adolescence (18-21 years) (reference: Bright Futures 3rd Edition, American Academy of Pediatrics. ${ }^{19}$ Placed in infanys were $22.7 \%$ of PICCs, $15.3 \%$ in early childhood, $16.2 \%$ in middle childhood, $23.3 \%$ in early adolescents, $16.8 \%$ in middle adolescents and $5.6 \%$ in late adolescents (see online supplementary table 1). At PICC insertion, the mean age was 8.7 years (IQR 1.7-14.6), weight was $33.5 \mathrm{~kg}$ (IQR 10-52) and height was $118.7 \mathrm{~cm}$ (IQR 76-159). Average PICC dwell time was 17.7 days (see online online supplementary table 2 ). 
The majority of PICCs $(97.8 \%)$ were placed by the ARNPs. Most were placed on a single attempt (79.6\%). PICC placements of $7.3 \%$ had $\geq 3$ attempts. The ultrasound guidance was used during $61.2 \%$ of PICC placement. The patient site of placement was right arm $(39.3 \%)$, left arm $(37.1 \%)$, right thigh (5\%), left thigh $(5.9 \%)$, right leg $(2.3 \%)$, left leg (2.6) and scalp (4.3\%). The tip of the PICC was confirmed to be in central circulation, as measured by plain radiography, in $92.7 \%$ of placements, with $77.9 \%$ in the SVC and $15 \%$ in the IVC. To confirm placement, the majority received one plain radiograph $(87.3 \%)$, while $\geq 3$ radiographs were obtained in $2.3 \%$ of patients. PICCs were placed in PICU (56.9\%), inpatient floor $(31.7 \%)$, paediatric specialty clinics $(5.4 \%)$ and operating room $(4.5 \%$ ) (see online supplementary table $1)$.

Single-lumen PICC lines were placed in $85.5 \%$ of patients, while double-lumen PICC lines were placed in $11.5 \%$ of patients. The majority of the patients had 3 French $(68.1 \%)$, 26 Gauge $(14.3 \%)$ or 4 French $(5.9 \%)$ (see online supplementary table 1). Approximately $75 \%$ of PICCs were placed for stable access, $9 \%$ for long-term antibiotics and $9 \%$ for chemotherapy. The majority of children $(58.7 \%)$ who received a PICC had at least one surgical procedure during their hospitalisation. Additionally, $14.6 \%$ of children who received a PICC had some underlying cardiac pathology. Overall, $11 \%$ of patients who received a PICC had an associated CVC at the time that the PICC was placed. Patients were sedated for PICC placement in $84.1 \%$ of cases, while $8.5 \%$ received both sedation and paralysis for PICC placement (see online supplementary table 1). The mean internal length of the PICC was $30.7 \pm 11.5 \mathrm{~cm}$, while the residual external length was $2.1 \pm 2.7 \mathrm{~cm}$ (see online supplementary table 2 ).

\section{Outcomes}

There were 49 CLABSIs (1.9\%) identified in the 2558 PICCs placed. The rate of CLABSI in infants and early childhood was higher than in those who were aged $\geq 5$ years $(2.8 \%$ and $3.1 \%$, respectively, vs $1.3 \%)$. In multivariate analysis after adjusting for the confounding effects of race and gender, infants (OR: 2.24, CI 1.14 to 4.39, $\mathrm{p}=0.02$ ) and those in early childhood (OR 2.37, CI 1.12 to $5.01, \mathrm{p}=0.02)$ had significantly higher odds of developing CLABSI compared with children $\geq 5$ years old (table 1 ).

Further evaluation of the infant subgroup demonstrated a $1.4 \%$ incidence of PICC-related rate and a

\begin{tabular}{|c|c|c|c|}
\hline Age group & OR & $95 \% \mathrm{Cl}$ & $P$ value \\
\hline Infants & 2.24 & 1.14 to 4.39 & 0.02 \\
\hline Early childhood & 2.37 & 1.12 to 5.01 & 0.02 \\
\hline$>5$ years of age & \multicolumn{3}{|c|}{ Reference } \\
\hline
\end{tabular}

Effects of race and sex were adjusted in the multivariable logistic regression model.

CLABSI, central line-associated blood stream infection.
CLABSI rate of $2.8 \%$. One patient required blood transfusion within 6 hours secondary to PICC-related bleeding. Detailed characteristics of the infants who received PICC in our study are described in the online supplementary table 3. In the unadjusted regression models (bivariate regression models), increase in number of days with PICC $(\mathrm{OR}=1.02,95 \% \mathrm{CI}=1.01$ to $1.03, \mathrm{p}=0.03)$, those who had an associated CVC at the time of PICC placement $(\mathrm{OR}=3.14,95 \% \mathrm{CI}=1.16$ to $8.54, \mathrm{p}=0.02)$, those needing a surgical procedure (surgical cohort) $(\mathrm{OR}=3.90,95 \%$ $\mathrm{CI}=1.10-$ to $3.83, \mathrm{p}=0.03)$ and those with a cardiac condition $(\mathrm{OR}=3.95,95 \% \mathrm{CI}=1.26$ to $12.41, \mathrm{p}=0.02)$ were associated with higher odds for developing CLABSI. However, in the multivariable regression analysis, none of these factors influenced the development of CLABSI in this age group (table 2). Multivariate logistic regression model was used to assess the impact of number of attempts to several patient and PICC-related factors. Infant age and topical analgesia use were associated with significantly higher number of attempts to place PICC successfully (table 3).

\section{Incidence and risk factors for developing CLABSI}

The rate of CLABSI was highest at $3.1 \%$ in early childhood cohort. There were 392 PICCs placed in this subgroup with the majority being male $(54.3 \%)$ and Caucasian (77.3\%). Most PICCs were placed in right upper extremity $(78.1 \%)$. Location of placement was PICU $(63.4 \%)$ and operating room (9\%). Mean external length of PICC was $2.2 \pm 2.6 \mathrm{~cm}$. Detailed characteristics of the infants who received PICC in our study are described in the online supplementary table 4 . Following adjustment for all available patient, provider, location and PICC confounders, the primary risk factor of CLABSI was the external length of the PICC. Each centimetre increase in length of PICC external (outside) to the skin was associated with a significantly higher odds for developing CLABSI (OR=1.30; $95 \% \mathrm{CI}=1.07$ to 1.57 ; $\mathrm{p}=0.008$ ). Interestingly, in this cohort, PICCs placed in the operating room were associated with significantly higher odds for developing CLABSI when compared with those that were placed in the PICU (OR=5.49, 95\% CI=1.03 to 29.19, $\mathrm{p}=0.04$ ) (table 4). The rate of thrombosis secondary to PICC placement was very low (1\%). Likewise, the rate of PICC-related bleeding that received blood transfusion within 6 hours was very low $(0.2 \%)$ (table 5 ).

\section{DISCUSSION}

\section{Overall outcomes of PICC placement in hospitalised children}

Our retrospective review of a large cohort of hospitalised children in whom PICCs were placed demonstrates that (1) an ARNP-driven PICC programme provides organisational efficiency as measured by success of PICC placement and (2) PICC-related complication rates including CLABSI, thrombosis and bleeding are low. Further, we show that children $<5$ years of age are more likely to require multiple attempts for successful PICC placement 
Table 2 Subset analysis of infant cohort (summary of estimates from the multivariable logistic regression model). risk of developing CLABSI

\begin{tabular}{|c|c|c|c|c|}
\hline \multirow[b]{2}{*}{ Variables } & \multirow[b]{2}{*}{ OR } & \multicolumn{2}{|l|}{$95 \% \mathrm{Cl}$} & \multirow{2}{*}{$\begin{array}{l}P \\
\text { value }\end{array}$} \\
\hline & & Lower & Upper & \\
\hline Age (in months) & 0.77 & 0.57 & 1.04 & 0.08 \\
\hline Male & 0.83 & 0.25 & 2.74 & 0.76 \\
\hline Upper extremity & 0.79 & 0.18 & 3.35 & 0.74 \\
\hline Scalp & 0.50 & 0.06 & 4.42 & 0.53 \\
\hline Lumen-double & 0.63 & 0.05 & 7.60 & 0.72 \\
\hline Number of attempts & 0.81 & 0.42 & 1.54 & 0.51 \\
\hline Surgical cohort & 4.80 & 0.93 & 24.92 & 0.06 \\
\hline Cardiac cohort & 1.23 & 0.27 & 5.69 & 0.79 \\
\hline $\begin{array}{l}\text { Presence of CVC at } \\
\text { PICC insertion }\end{array}$ & 2.12 & 0.63 & 7.11 & 0.22 \\
\hline $\begin{array}{l}\text { Length external to skin } \\
\text { (length outside) in } \mathrm{cm}\end{array}$ & 1.10 & 0.93 & 1.32 & 0.27 \\
\hline $\begin{array}{l}\text { Length internal (inside) } \\
\text { in } \mathrm{cm}\end{array}$ & 1.03 & 0.92 & 1.16 & 0.59 \\
\hline Caucasian & 1.44 & 0.28 & 7.32 & 0.66 \\
\hline $26 \mathrm{G}$ & 0.25 & 0.07 & 0.90 & $0.03^{*}$ \\
\hline PICC days & 1.01 & 0.99 & 1.03 & 0.29 \\
\hline
\end{tabular}

*statastically significant

CLABSI, central line-associated bloodstream infection; CVC, central venous catheter; PICC, peripherally inserted central catheter.

and are twice as likely to develop CLABSIs. Risk factors associated with developing CLABSIs in this younger population include the presence of residual catheter length 'outside (external)' to the skin and the placement of these PICCs in the operating room.

\section{Impact of ARNP-directed vascular access/PICC programmes}

Vascular access ARNP-driven PICC programmes are well established in premature neonates and adults. A survey of 187 neonatal intensive care unit (NICU) demonstrates that ARNPs are primarily responsible for PICC placement in $74.3 \%(139 / 187)$ of programme. ${ }^{20}$ Similarly, a singlecentre study describes a nurse-led programme that successfully placed bedside PICCs in $74 \%$ of adult oncology patients to allow $95 \%$ of those patients to complete prescribed chemotherapy treatment. ${ }^{21}$ Similar literature in paediatrics is limited largely to smaller, single-centre studies. A prospective single-centre study has described a nurse-led PICG programme that successfully placed PICC in $71.1 \%$ (69/99) of children. ${ }^{22}$ However, this high rate of success may have been exaggerated as only cooperative children $>3$ year of age were included, and PICCs were placed in the interventional radiology suites to confirm placement by fluoroscopy. ${ }^{22}$ Our vascular ARNPdriven PICC programme was very effective and placed the majority (97.8\%) of 2558 PICCs in our institutions over a 7-year period, with $79.6 \%$ of those on the first attempt.
In addition to team efficacy in line placement, a number of studies have demonstrated lower complication rates with nurse-lead PICC programme. McDiarmid et al have reported successful implementation of an advanced practice nurse-led team in adults with low rates of PICC-related complications. ${ }^{23}$ Our study also demonstrated low rates of CLABSI, thrombosis and acute bleeding needing transfusion. Our CLABSI rate of $2 \%$ during the study period is consistent with current reported prevalence of CLABSI in neonates and children $(1.3 \%-2.6 \%) .{ }^{24}$ However, this incidence is twofold higher in children $<5$ years. Given the Joint Commission's 2010 National Patient Safety Goal of reducing CLABSI rates, ${ }^{1}$ we further assessed this subgroup of patients and were able to show that both the residual external catheter length and the placement of PICCs in the operating room were associated with increased risk of developing CLABSI.

\section{Higher risk on CLABSI in those who had their PICCs placed in the operating room}

In our study, less than $10 \%$ of all PICCs were placed in the operating room. Children between 1 and 5 years of age, who had a PICCs placed in the operating room were more likely to develop CLABSI. While these data are surprising, we believe there are several potential explanations. Our ARNP team places PICCs using a formal, well-established insertion bundle. The presence of insertion bundles is known to decreases CLABSIs. ${ }^{5}{ }^{6}$ It is unclear whether placement of PICCs in the operating room followed such procedures, particularly since less than $10 \%$ of all PICCs were placed in the operating room and, therefore, less routine. Further, it is possible that those patients who had PICCs placed in the operating room were more acutely ill in comparison to those patients who had PICCs placed in other locations. Unfortunately, given the retrospective nature of this study, we were unable to assess severity of illness for these patients. Further studies are needed to confirm these findings.

\section{Impact of catheter length and CLABSI}

Our data that residual 'external' catheter length outside the skin is associated with increased incidence of CLABSI in children $<5$ years is a new finding. Many institutional protocols emphasise minimising dislodgement of the catheter, which accounts in part for the rationale of leaving some amount of catheter external to the skin. However, the possibility of spontaneous catheter migration and micromovements during catheter maintenance may explain the increase in infection. There are several reports of PICCs migrating in younger children and leading to complications, ${ }^{25-27}$ such spontaneous catheter migration is less frequently reported in older children, adolescents or adults. PICC migration may occur during catheter maintenance secondary to PICC-related factors such as smaller diameter catheter or patient-related factors such as movement during dressing change or change in patient condition (ie, oedema, skin integrity). The increased risk of CLABSI with residual external 
Table 3 Summary of estimates from multivariable linear regression model to examine the number of attempts to obtain a successful PICC

$95 \% \mathrm{Cl}$

\begin{tabular}{lllll} 
Independent variables & Parameter estimate & Lower bound & Upper bound & P value \\
\hline Age group & & & & \\
Infancy: 0-12 months & Reference group & & & \\
\hline Early childhood: $13-59$ months & -0.453 & -0.555 & -0.351 & $<0.0001^{*}$ \\
\hline Middle childhood: $60-131$ months & -0.546 & -0.65 & -0.442 & $<0.0001^{*}$ \\
\hline Early adolescence: 132-179 months & -0.602 & -0.703 & -0.502 & $<0.0001^{*}$ \\
\hline Middle adolescence: 180-215 months & -0.554 & -0.662 & -0.447 & $<0.0001^{*}$ \\
\hline Late adolescence: 216-251 months & -0.608 & -0.755 & -0.462 & $<0.0001^{*}$ \\
\hline Covariates & & & & \\
\hline Male & -0.002 & -0.057 & 0.054 & 0.954 \\
\hline Caucasian & 0.075 & -0.003 & 0.153 & 0.061 \\
\hline Place-general floor & -0.06 & -0.127 & 0.007 & 0.08 \\
\hline Place-operating room & -0.116 & -0.255 & 0.023 & 0.103 \\
\hline Place-specialty clinic & -0.086 & -0.216 & 0.043 & 0.192 \\
\hline Place-burns unit & 0.171 & -0.271 & 0.614 & 0.448 \\
\hline Place-other & 0.385 & -0.013 & 0.783 & 0.058 \\
\hline Presence of CVC at PICC insertion & -0.039 & -0.134 & 0.056 & 0.425 \\
\hline USG-used & -0.021 & -0.081 & 0.039 & 0.499 \\
\hline Topical analgesia-used & -0.117 & -0.18 & -0.053 & $<0.0001^{*}$ \\
\hline Sedation used & -0.004 & -0.082 & 0.074 & 0.913 \\
\hline Paralysis used & 0.097 & -0.01 & 0.205 & 0.077 \\
\hline Surgical cohort & 0.019 & -0.04 & 0.078 & 0.526 \\
\hline Cardiac cohort & 0.089 & -0.004 & 0.182 & 0.06 \\
\hline
\end{tabular}

*statastically significant

CVC, central venous catheter; PICC, peripherally inserted central catheter; USG, ultrasound.

catheter length in younger children may have practical implications, including more specific determination of catheter length at the time of insertion and more careful attention to residual catheter length during catheter maintenance. This is the first study to our knowledge that demonstrates significantly higher odds of developing CLABSI with increased length in centimetres of residual external catheter length at insertion.

\section{PICCs and risk of thrombosis}

Another significant central venous access-related morbidity is thrombosis. ${ }^{11}$ The incidence of catheter-related thrombosis has increased significantly over the past decade. ${ }^{17}$ This may be secondary to the improved care of paediatric patients with serious disorders, prolonged survival rates in children with chronic diseases and advancement in diagnostic techniques. ${ }^{17}$ The reported prevalence of catheter-related thrombosis varies from $1 \%$ to $9 \%$ in critically ill children. ${ }^{1728}$ One study demonstrated the thrombosis prevalence is lower with PICCs and not significantly different when comparing upper and lower extremities. ${ }^{29}$ In our study, PICC-related thrombosis rate was low $(1 \%)$ consistent with the published literature.
During the study period, diagnostic vascular ultrasound was obtained at the discretion of treating physician indicating that the PICC-related thrombosis was symptomatic. Similarly, PICC-related bleeding rates were extremely low suggestive of safety of the instruments and devices used during placement and provider adherence to safety protocols.

\section{Number of attempts to successfully obtain a PICC and related outcomes}

Providers aim for successful PICC placement in the least number of attempts. Greater than one attempt is an independent predictor of PICC-related thrombosis in adult oncology patients. ${ }^{30}$ Further, minimal access attempts are associated with greater patient satisfaction. $^{18}$ Multiple patient-related variables including small vein size, anatomic variations in vasculature and suboptimal patient cooperation may result in difficulty in achieving intravenous access. In this study, the ARNPs successfully placed the majority of PICCs $(97.8 \%)$, and most of the PICCs were placed in a single attempt $(79.6 \%)$. Unsurprisingly, we found that infants required significantly more attempts for successful 
Table 4 Subset analysis of early childhood cohort (summary of estimates from the multivariable logistic regression model). risk of developing CLABSI

\begin{tabular}{|c|c|c|c|c|}
\hline Variables & OR & Lower $95 \%$ & Upper $95 \%$ & $P$ value \\
\hline Age (each 1 month increase) & 0.98 & 0.93 & 1.04 & 0.49 \\
\hline Male & 0.57 & 0.14 & 2.30 & 0.43 \\
\hline Caucasian & 0.49 & 0.11 & 2.18 & 0.35 \\
\hline \multicolumn{5}{|l|}{$\begin{array}{l}\text { Hospital location/place where PICC was placed: } \\
\text { PICU (reference Pediatric Intensive Care Unit) }\end{array}$} \\
\hline Place-general floor & 0.66 & 0.06 & 7.03 & 0.73 \\
\hline Place-operating room & 5.49 & 1.03 & 29.19 & $0.04^{*}$ \\
\hline Place-specialty clinic & 2.90 & 0.26 & 32.79 & 0.39 \\
\hline Place-burns unit & $\mathrm{DNC}^{*}$ & & & \\
\hline Place-other & $\mathrm{DNC}^{*}$ & & & \\
\hline Presence of CVC at PICC insertion & 0.77 & 0.07 & 8.23 & 0.83 \\
\hline Ultrasound used & 2.00 & 0.43 & 9.35 & 0.38 \\
\hline Lumens & 0.81 & 0.06 & 10.44 & 0.87 \\
\hline Length external to skin (length outside) in $\mathrm{cm}$ & 1.30 & 1.07 & 1.57 & $0.008^{*}$ \\
\hline Length internal (inside) in $\mathrm{cm}$ & 1.08 & 0.93 & 1.26 & 0.29 \\
\hline Surgical cohort & 0.50 & 0.12 & 2.06 & 0.34 \\
\hline Cardiac cohort & 5.33 & 0.46 & 62.00 & 0.18 \\
\hline Number of site attempts & 1.51 & 0.61 & 3.75 & 0.38 \\
\hline PICC days & 1.00 & 0.98 & 1.02 & 0.93 \\
\hline
\end{tabular}

${ }^{*}$ DNC-Model did not converge since events are too low.

CLABSI, central line-associated bloodstream infection; PICC, peripherally inserted central catheter; PICU, paediatric intensive care unit.

PICC placement. Children who received topical analgesia required fewer number of attempts for successful PICC placement when compared with those who did not receive topical analgesia. Use of real-time ultrasound guidance has become the standard of practice for CVC placement and decreases overall number of attempts with significantly lower complication rates. ${ }^{29}$ Use of ultrasound guidance for successful PICC placement is well established in adults. ${ }^{31}{ }^{32}$ However, there are limited published data to support using vascular ultrasound for PICG placement in children. In one small, prospective randomised single-centre trial, the use of ultrasound in 21 patients undergoing PICC placement with median age of 2.3 years showed significant success on first attempt when compared with the non-ultrasound control group ( $90.5 \%$ vs $47.6 \%, p=0.03$ ). Ultrasound guidance enhances the provider's ability to access deeper, non-visible, non-palpable vessels such as basilic, cephalic or femoral veins especially in younger ages. ${ }^{33}$ In our study, ultrasound was used for PICC placement in

Table 5 Overall PICC-related complications during the study period

\begin{tabular}{|c|c|c|c|}
\hline Variable & Characteristic & $\begin{array}{l}\text { Overall } \\
\mathrm{N}(\%)\end{array}$ & $\begin{array}{l}\text { Early childhood } \\
\mathrm{N}(\%)\end{array}$ \\
\hline \multirow[t]{2}{*}{ CLABSI associated with the PICC* } & Yes & 49 (1.9) & $12(3.1)$ \\
\hline & No & $2453(95.9)$ & $371(94.6)$ \\
\hline \multirow[t]{2}{*}{ Thrombosis associated with the PICC $†$} & Yes & $25(1)$ & $3(0.8)$ \\
\hline & No & $2461(96.2)$ & $379(96.7)$ \\
\hline \multirow[t]{2}{*}{ Significant bleeding associated with the PICC proceduref } & Yes & $6(0.2)$ & - \\
\hline & No & 2499 (97.7) & $383(97.7)$ \\
\hline
\end{tabular}

${ }^{*}$ As per institutional definition of CLABSI, based on CDC guidelines.

†Thrombosis diagnosed by vascular ultrasound and confirmed by radiologist at the site of PICC placement within 3 months of PICC discontinuation.

$¥$ Significant bleeding was defined as those that needed blood transfusion within 6 hours after placing the PICC.

CDC, Centers for Disease Control and Prevention; CLABSI, central line-associated bloodstream infection; PICC, peripherally inserted central catheter. 
approximately two-thirds of PICC attempts, which likely contributed to our overall success in placement.

In our cohort, the majority of PICCs were placed in the upper extremities; however, the younger age group had more PICCs placed in the lower extremities. After adjusting for the potential confounders, there was no significant difference noted in outcomes (CLABSI) with various sites of insertion (laterality: right vs left side, upper vs lower extremity) of PICC placement. This information may help providers during informed consent process.

\section{Limitations of our study}

Several considerations should be made when interpreting this study. This is a retrospective study that is based on availability of data elements and inability to adjust for unknown confounders. We excluded PICCs that were primarily placed in NICU by their providers and those that were placed at outside institutions to minimise the impact of the provider, unit and institutional variances. Additionally, we excluded those patients that were discharged with a PICC in place as follow-up was limited after hospital discharge. However, while paediatric patients were rarely discharged to home with a PICC in place during the study period, this may have excluded a subpopulation that had a greater severity of illness, an increased length of stay or were discharged on prolonged antibiotic therapy for blood stream infection. Although our central line catheter insertion and maintenance bundles were formally standardised and adhered to, it is possible that such practices could have been breached during the study period. In our institution, all CLABSIs were reviewed extensively for any breach in such standards, and we were unable to identify obvious deviation in practices. These institutional catheter maintenance practices remained unchanged throughout the study period. An additional important limitation is that we were not able to adjust for severity of illness in assessing CLABSI rates. In this study, we used the number of CLABSI events rather than the incidence rate (CLABSI per 1000 catheter days) due to the statistical limitation of the analysing the low CLABSI rates overall. Finally, despite the large cohort, this is a single-institution study, and these findings may not be generalisable.

\section{Implications for clinical practice and future directions}

PICCs are an integral part of providing care for hospitalised paediatric patients. A well-organised vascular access ARNP-driven PICC programme can successfully optimise care in hospitalised paediatric patients. The majority of PICCs were successfully placed by ARNPs, within the first attempt. Infants required more attempts for successful PICC placement. Overall, the success rate of PICC placement is high and the complication rate is low. An increase in complication rates with younger age, residual catheter length in centimetres outside the skin, and placement in the operating room requires further confirmation in a multicentre study. No significant difference between upper versus lower extremity versus scalp or laterality (right vs left vs scalp) in PICG placement or its associated complications was noted in our study. Further research is needed to evaluate the impact of certain factors such as measurement techniques on minimising residual catheter length in centimetres outside the skin and decreasing CLABSI rates, as well as other outcomes that were evaluated.

\section{CONCLUSIONS}

Our findings suggested that younger children $(<5$ years of age) are at an increased risk of developing PICC related CLABSIs. Residual external catheter length in centimetres outside the skin and PICC placement in the operating room are independent predictors of CLABSI in those children between 1 and 5 years of age. PICC-related thrombosis and bleeding are infrequent complications. Our findings may have clinical implications and can be used to tailor PICC placement and the development of ARNP led vascular access teams, though further prospective multicentre studies are needed to confirm these findings.

Negative estimates imply fewer number of attempts. Likewise, positive estimates imply higher number of attempts. For example, in the regression table, the variable 'topical analgesia used' has a negative estimate of -0.117 ( -0.18 to $-0.053 ; 95 \%$ CIs do not cross to positive) with a $\mathrm{p}$ value that is significant $(<0.0001)$. This means that those patients who received topical analgesia had fewer number of attempts at successful PICC placement when compared with those who did not receive topical analgesia. Similarly, early childhood, middle childhood, early or middle or late adolescence children had fewer number of attempts at successful placement of PICCs compared with infants (ie, infants had higher number of attempts at successful placement of PICCs).

\section{Author affiliations}

${ }^{1}$ Pediatrics, University of lowa Children's Hospital, lowa City, Lowa, USA

${ }^{2}$ Management Sciences, University of lowa Henry B Tippie College of Business, lowa City, Lowa, USA

${ }^{3}$ Health Care Administration, Rhode Island College, Providence, Rhode Island, USA ${ }^{4}$ Division of Pediatric Critical Care, Department of Pediatrics, Duke Children's Hospital and Health Center, Durham, North Carolina, USA

${ }^{5}$ Department of Orthodontics, University of Illinois at Chicago, Chicago, Illinois, USA

Acknowledgements University of lowa Hospital Quality and Infection control division for providing access to CLABSI data. Dr. Katharine Robb, MD for technical support.

Contributors Conceptualisation: VjA, AB, MA. Data curation: AB, JB, AS, BF, TW. Formal analysis: VjA, SR, VsA, TW. Investigation: VjA, SR, VsA, TW. Methodology: VjA, SR, VsA, MA, JB, AB, AS, BF, TW. Project administration: VjA, AB, VsA, MA, JT. Resources: VjA, VsA, MA, JT. Software: SR, VsA, TW. Supervision: VjA, VsA, MA, JT. Validation: VjA, SR, VsA, TW. Writing —original draft: AB, VjA, SR, VsA, MA, JB, AS, BF, TW. Writing — review and editing: AB, VjA, SR, VsA, MA, JB, AS, BF, TW, JT. All authors are represented by their respective initials.

Funding Aditya Badheka, MD, MS was the recipient of a travel award for presenting the results of this study at the 47th Congress, SCCM, San Antonio, February 2018.

Competing interests None declared.

Patient consent for publication Not required.

Provenance and peer review Not commissioned; externally peer reviewed. 
Data availability statement All data relevant to the study are included in the article or uploaded as supplementary information.

Open access This is an open access article distributed in accordance with the Creative Commons Attribution Non Commercial (CC BY-NC 4.0) license, which permits others to distribute, remix, adapt, build upon this work non-commercially, and license their derivative works on different terms, provided the original work is properly cited, appropriate credit is given, any changes made indicated, and the use is non-commercial. See: http://creativecommons.org/licenses/by-nc/4.0/.

\section{REFERENCES}

1. Advani S, Reich NG, Sengupta A, et al. Central line-associated bloodstream infection in hospitalized children with peripherally inserted central venous catheters: extending risk analyses outside the intensive care unit. Clin Infect Dis 2011;52:1108-15.

2. Marnejon T, Angelo D, Abu Abdou A, et al. Risk factors for upper extremity venous thrombosis associated with peripherally inserted central venous catheters. J Vasc Access 2012;13:231-8.

3. Wilson TJ, Brown DL, Meurer WJ, et al. Risk factors associated with peripherally inserted central venous catheter-related large vein thrombosis in neurological intensive care patients. Intensive Care Med 2012;38:272-8.

4. Jumani K, Advani S, Reich NG, et al. Risk factors for peripherally inserted central venous catheter complications in children. JAMA Pediatr 2013;167:429-35.

5. Pronovost $\mathrm{P}$, Needham D, Berenholtz S, et al. An intervention to decrease catheter-related bloodstream infections in the ICU. N Engl J Med 2006;355:2725-32.

6. Miller MR, Griswold M, Harris JM, et al. Decreasing PICU catheterassociated bloodstream infections: NACHRI's quality transformation efforts. Pediatrics 2010;125:206-13.

7. Prevention CoDCa, 2016. Available: www.cdc.gov/HAl/pdfs/ progress-report/hai-progress-report.pdf [Accessed 21 May 2018].

8. Garland JS, Alex CP, Sevallius JM, et al. Cohort study of the pathogenesis and molecular epidemiology of catheter-related bloodstream infection in neonates with peripherally inserted central venous catheters. Infect Control Hosp Epidemiol 2008;29:243-9.

9. García-Teresa MA, Casado-Flores J, Delgado Domínguez MA, et al. Infectious complications of percutaneous central venous catheterization in pediatric patients: a Spanish multicenter study. Intensive Care Med 2007;33:466-76.

10. Sengupta A, Lehmann C, Diener-West M, et al. Catheter duration and risk of CLA-BSI in neonates with PICCs. Pediatrics 2010;125:648-53.

11. Rooden CJ, Tesselaar MET, Osanto S, et al. Deep vein thrombosis associated with central venous catheters - a review. J Thromb Haemost 2005;3:2409-19.

12. Evans RS, Sharp JH, Linford LH, et al. Risk of symptomatic DVT associated with peripherally inserted central catheters. Chest 2010;138:803-10.

13. Liem TK, Yanit KE, Moseley SE, et al. Peripherally inserted central catheter usage patterns and associated symptomatic upper extremity venous thrombosis. J Vasc Surg 2012;55:761-7.

14. Evans RS, Sharp JH, Linford LH, et al. Reduction of peripherally inserted central catheter-associated DVT. Chest 2013;143:627-33.

15. Cook D, Crowther M, Meade M, et al. Deep venous thrombosis in medical-surgical critically ill patients: prevalence, incidence, and risk factors. Crit Care Med 2005;33:1565-71.
16. Tran H, Arellano M, Chamsuddin A, et al. Deep venous thromboses in patients with hematological malignancies after peripherally inserted central venous catheters. Leuk Lymphoma 2010;51:1473-7.

17. Kanin M, Young G. Incidence of thrombosis in children with tunneled central venous access devices versus peripherally inserted central catheters (PICCs). Thromb Res 2013;132:527-30.

18. Costantino TG, Parikh AK, Satz WA, et al. Ultrasonography-guided peripheral intravenous access versus traditional approaches in patients with difficult intravenous access. Ann Emerg Med 2005;46:456-61.

19. American Academy of Pediatrics. Bright futures edition 3. Available: https://brightfutures.aap.org/Pages/default.aspx [Accessed 6 Jun 2019].

20. Sharpe E, Pettit J, Ellsbury DL. A national survey of neonatal peripherally inserted central catheter (PICC) practices. Adv Neonatal Care 2013;13:55-74.

21. Barber JM, Booth DM, King JA, et al. A nurse led peripherally inserted central catheter line insertion service is effective with radiological support. Clin Radiol 2002;57:352-4.

22. Gamulka B, Mendoza C, Connolly B. Evaluation of a unique, nurseinserted, peripherally inserted central catheter program. Pediatrics 2005;115:1602-6.

23. McDiarmid S, Scrivens N, Carrier M, et al. Outcomes in a nurse-led peripherally inserted central catheter program: a retrospective cohort study. CMAJ Open 2017;5:E535-9.

24. Safdar N, Maki DG. Risk of catheter-related bloodstream infection with peripherally inserted central venous catheters used in hospitalized patients. Chest 2005;128:489-95.

25. Orme R M L'E, McSwiney MM, Chamberlain-Webber RFO. Fatal cardiac tamponade as a result of a peripherally inserted central venous catheter: a case report and review of the literature. $\mathrm{Br} J$ Anaesth 2007;99:384-8.

26. Sertic AJ, Connolly BL, Temple MJ, et al. Perforations associated with peripherally inserted central catheters in a neonatal population. Pediatr Radiol 2018;48:109-19.

27. Sharpe E, Kuhn L, Ratz D, et al. Neonatal peripherally inserted central catheter practices and providers: results from the neonatal PICC1 survey. Adv Neonatal Care 2017;17:209-21.

28. Gasior AC, Marty Knott E, St Peter SD. Management of peripherally inserted central catheter associated deep vein thrombosis in children. Pediatr Surg Int 2013;29:445-9.

29. Duesing LA, Fawley JA, Wagner AJ. Central venous access in the pediatric population with emphasis on complications and prevention strategies. Nutr Clin Pract 2016;31:490-501.

30. Jones D, Wismayer K, Bozas $\mathrm{G}$, et al. The risk of venous thromboembolism associated with peripherally inserted centra catheters in ambulant cancer patients. Thromb J 2017;15:25.

31. Stokowski G, Steele D, Wilson D. The use of ultrasound to improve practice and reduce complication rates in peripherally inserted central catheter insertions: final report of investigation. J Infus Nurs 2009;32:145-55.

32. Nichols I, Humphrey JP. The efficacy of upper arm placement of peripherally inserted central catheters using bedside ultrasound and microintroducer technique. J Infus Nurs 2008;31:165-76.

33. Sandhu NPS, Sidhu DS. Mid-arm approach to basilic and cephalic vein cannulation using ultrasound guidance. $\mathrm{Br} J$ Anaesth 2004;93:292-4 